\title{
La implicación parental según los estilos educativos y el género de los padres
}

\author{
ENRIC LLIN VAÑÓ \\ llin@uji.es \\ Clara Renau Escrig \\ crenau@uji.es \\ SARA RoselLo SEMPERE \\ srosello@uji.es \\ GHISLAINE MARANDE PERRIN \\ marande@uji.es \\ FRANCISCO JUAN GaRcía BACETE \\ fgarcia@uji.es
}

\section{Resumen}

Introducción: El estudio de la implicación parental es vital durante las primeras etapas de la educación primaria ya que se derivan numerosos beneficios del trabajo conjunto entre familia y escuela. Es importante conocer que factores influyen en la implicación en estas etapas como las creencias, normas culturales, el tipo de familia, el nivel cultural, el género de los padres. A través de una investigación cuantitativa, el presente trabajo estudia si los estilos educativos y el género de los padres influyen en diversos aspectos de la implicación parental como la frecuencia de contacto entre la familia, la calidad de las relaciones entre padres y profesores, la implicación y voluntariado de los padres en la escuela o el apoyo de los padres a los hijos. Método: 195 familias de niños/as de $1^{\circ}$ de primaria contestaron el cuestionario «Parent-Teacher Involvement» (PTI) y «Parent Styles and Dimension Questionarie» (PSDQ). Resultados: No se cumplen las hipótesis ya que los estilos educativos y el género no influyen significativamente en la implicación parental aunque si se obtuvieron diferencias significativas entre los estilos educativos de padres y madres. Por otra parte, el $88,2 \%$ de los cuestionarios fueron contestados por madres lo que respalda afirmaciones como que las madres son el principal vínculo entre el hogar y la escuela.

Palabras clave: Implicación parental, estilos educativos, familia y escuela.

\section{Abstact}

Introduction: This report studies parental involvement, which is very important in early primary education stages because many benefits of joint work between family and school 
result. Knowing which factors influence involvement in these stages is relevant; e.g., beliefs, cultural norms, family type, cultural level or parents' gender. A quantitative research examined whether educational styles and parents' gender influenced parental involvement in areas such as frequency of parent-teacher contact, quality of Parent/Teacher Relationships, Parents' involvement and Volunteering at School and Parents' Endorsement of the Child's School. Method: The «Parent Teacher Involvement» (PTI) questionnaire and «Parent Dimensions Styles and Questionnaire» (PSDQ) were answered by 195 families of year-1 primary education schoolchildren. Results: The main hypothesis was not met because no significant relationship existed between educational styles and parental involvement. Moreover, $88.2 \%$ of the questionnaires were answered by mothers, which thus supports the notion that mothers are still the main link between family and school.

Keywords: parental involvement, educational styles, family, school.

\section{Introducción}

\section{Implicacion parental}

La implicación parental se ha constatado como un aspecto particularmente importante porque tiene repercusiones significativas para la educación de los niños (Mattingly, Prislin, McKenzie, Rodriguez y Kayzar, 2002). Diversos estudios empíricos han demostrado la conexión entre la calidad de las relaciones familia - escuela y los resultados positivos en términos académicos, en habilidades socioemocionales y problemas de conducta, particularmente durante la infancia temprana (Iruka, Winn, Kingsely, y Orthodoxou, 2011; Powell y Cols., 2010; RimmKaufman, Pianta, Cox, y Bradley, 2003; Serpell y Mashburn, 2011). Otros tipos de estudios han identificado factores que influyen en la participación y en sus efectos como las creencias, normas culturales, atributos ambientales (Harkness y Súper, 1996) o el género de los padres (Pleck y Masciadrelli, 2004).

Aunque Keith y Lichtman (1994) afirman que la implicación parental es un término vago con multitud de significados, Epstein (2001) desarrolló un marco, para definir la implicación parental, basándose en diversas actividades en las que pueden estar implicadas las familias. En concreto, se establece seis tipos de participación: la parentalidad (obligaciones de la familia en el hogar), la comunicación (intercambio de información sobre el progreso educativo de los estudiantes), voluntariado (participación de los padres en las actividades escolares), el aprendizaje en casa, la toma de decisiones, y la colaboración con la comunidad. Según Hornby (2011) la implicación parental se puede manifestar a través de varias formas como: actividades informales que incitan a los padres a asistir a la escuela, diferentes formatos de reuniones entre los padres y el profesorado, y otras formas de comunicación como los contactos telefónicos, y con menos frecuencia, la opción de las nuevas tecnologías o las visitas a casa.

La implicación parental se da, principalmente, por parte de las madres (Esquivel, 1995). Sobre todo la implicación de las madres es superior cuando hablamos de familias tradicionales o biológicas (Harris, Furstenberg y Marmer 1998; Nord, Brimhall y West 1997). Henderson y Mapp (2002) afirman que los padres y las madres se implican de forma igualitaria en la educación de sus hijos pero en la escuela las madres se implican más que los padres. 
Diversos estudios justifican el estudio temprano de la implicación parental porque tiende a disminuir a final de primaria y en los primeros años de la escuela secundaria. (Adams y Christenson, 2000; Grolnick y Cols., 2000; McCaslin y Murdock, 1991; Simon, 2004). Shaver y Walls (1998) afirma la implicación era superior cuando los niños estaban en la escuela, en el instituto o la universidad, por esta razón consideramos importante estudiar cómo se desarrolla la implicación parental en primero de primaria.

\section{Factores que influyen en la Implicación de los padres}

Los investigadores se han preguntado acerca de muchas cuestiones relevantes sobre cómo es la implicación parental, ¿Por qué ciertos padres se involucran y otros no? ¿Qué motiva a las personas a participar? ¿Por qué la implicación parental es beneficiosa para los estudiantes? Hay estudios que afirman la existencia de aspectos que influyen en la implicación parental como el tipo de familia (biológica, monoparental, reconstituida,..) (National center for education statistics, 2001) o el género de los padres/madres (Hsu y Cols. 2011; Ho y cols., 2010; Hung, 2005) pero nadie ha estudiado si los estilos educativos afectan a la implicación. Uno de los principales motivadores de la participación según Hoover-Dempsey y cols. (2005) es la construcción del rol parental y puede definirse como las creencias que los padres tienen sobre las actuaciones en relación a la educación de sus hijos.

\section{Estilos educativos}

Baurmind clasifica los estilos educativos en autoritativo, autoritario y permisivo. El estilo autoritativo se caracteriza por un nivel alto de afecto y comunicación, como de control y exigencia. El estilo autoritario se caracteriza por un alto control, rigidez y exigencias frente a la escasez de afecto y comunicación. El estilo permisivo se caracteriza por altos niveles de afecto y comunicación pero bajos niveles de control y exigencia (Comino y Raya, 2014).

Por otra parte, Broderick y Blewitt (2006) afirman que los estilos educativos tienen efectos dominantes sobre la conducta de aprendizaje del niño, siendo el comportamiento de los padres muy importante para el futuro de la educación de su hijo. El paralelismo de definiciones entre Estilos educativos (Broderick y Blewitt, 2006) y la Construcción del rol parental (Hoover-Dempsey y cols., 2005) y la ausencia de estudios que relacionen los estilos educativos con la implicación parental le proporciona a este tema de estudio la importancia para llevarse a cabo.

Algunos investigadores tienen la teoría de que las mujeres pueden estar biológicamente preparadas (Rossi, 1978) o tener socialmente inculcado (Downey 1994) una forma de ser más enriquecedora que los hombres. La pretensión es que las mujeres son socializadas para satisfacer las necesidades expresivas de los niños, para ser comunicadoras, y mostrar calor y afecto (Thomson, McLanahan, y Curtin 1992). Los hombres, por otro lado, se les enseña a ser los responsables de la familia, encargados de hacer cumplir las reglas y ser disciplinarios. Esto sugiere que los hombres y las mujeres realizan diferentes funciones dentro de la familia (Thomson, McLanahan, y Curtin 1992). Esto induce a pensar que los estilos educativos adoptados por padres y madres serán diferentes 


\section{Objetivos e hipótesis}

El objetivo principal de este trabajo es estudiar si los estilos educativos y el género de los padres influyen en la implicación parental en alumnos de primero de primaria. Y nuestras hipótesis serán dos: que los estilos educativos influyen en la implicación parental y que la implicación de las madres será superior a los padres.

\section{Método}

\section{Participantes}

La muestra estudiada se obtuvo a través del grupo GREI (Grupo interuniversitario del estudio del rechazo entre iguales). Este equipo está dividido dentro del territorio Español en Castelló de la Plana, Sevilla, Palma de Mallorca y Valladolid. De la muestra total que utilizó el equipo GREI, se seleccionó una submuestra con la finalidad de obtener información de sus familias. Esta estuvo formada por 195 familias de niños y niñas de primero de primaria a las cuales se les entregó un sobre con determinados cuestionarios.

\section{Instrumentos de medida}

Los cuestionarios subministrados a las familias fueron el Parent Teacher Involvement» (PTI) y el «Parent Styles and Dimension Questionarie» (PSDQ).

Implicación de los padres en la escuela. El cuestionario PTI está compuesto por una escala Likert de 0 a 4 siendo 0 nunca y 4 más de una vez por semana. Los factores sobre los que trabaja este cuestionario son: la frecuencia de contacto entre la familia y la escuela, la calidad de las relaciones entre padres y profesores, la implicación y voluntariado de los padres en la escuela, el apoyo de los padres a los hijos. Los factores del cuestionario PTI se asemejan a los tipos de implicación parental definidos por Epstein de «Comunicación», «Parentalidad», «Voluntariedad» y «Aprendizaje en casa».

Estilos educativos de los padres. El cuestionario PSDQ está compuesto por una escala Likert del 1 al siendo 1 nunca y 5 siempre. Este cuestionario se utiliza para determinar los estilos educativos de los padres y madres, distinguiendo entre tres estilos diferentes: los autoritarios, autoritativos y permisivos.

La recogida de datos se realizó mediante la escuela que le entregó y recogió a cada familia los cuestionarios correspondientes. Los cuestionarios fueron transcritos al programa de análisis estadístico IBM SPSS Statistics Base Versión 21, en el que también se realizaron los análisis.

\section{Estrategias de análisis}

Antes de realizar los análisis y la factorización de los cuestionarios se realizó la depuración de la matriz, seleccionando la submuestra implicada en el estudio y tratando los valores perdidos. Una vez hecho este trabajo realizamos un conglomerado bietápico para obtener los estilos educativos de cada sujeto. 
Finalmente realizamos los estadísticos descriptivos para ver el estilo educativo, pruebas T para muestras independientes y análisis de la varianza (ANOVA). Estos resultados se presentan a continuación.

\section{Resultados}

A continuación se presentan los resultados obtenidos. Los estadísticos descriptivos mostraron que de 195 familiares que contestaron el PTI un $88,2 \%$ fueron madres y un $11,8 \%$ padres. Para determinar las agrupaciones en los estilos educativos de madres y padres se realizó un análisis de conglomerados bietápico (Tablas 1 y 2). En las madres, a partir de 3 entradas, se obtuvieron 2 conglomerados con una calidad del conglomerado de 0,5 en la medida de silueta de cohesión y separación. Siendo el conglomerado Autoritativo del 56,4\% (97) y el Autoritario/ Pasivo del $43,6 \%$ (75).

Tabla 1

Conglomerado bietápico Factores Robinson Madres

\begin{tabular}{lcccccc}
\hline \multirow{2}{*}{$\begin{array}{l}\text { Conglomerados } \\
\text { Robinson Madres }\end{array}$} & \multicolumn{2}{c}{$\begin{array}{c}\text { Factor Robinson } \\
\text { Autoritativo }\end{array}$} & \multicolumn{2}{c}{$\begin{array}{c}\text { Factor Robinson } \\
\text { Autoritario }\end{array}$} & \multicolumn{2}{c}{$\begin{array}{c}\text { Factor Robinson } \\
\text { Permisivo }\end{array}$} \\
\cline { 2 - 7 } & Media & DT & Media & DT & Media & DT \\
\hline Autoritativo & 4,43 &, 28 & 1,77 &, 20 & 1,56 &, 18 \\
Autoritario/Permisivo & 4,04 &, 47 & 2,33 &, 29 & 1,90 &, 28 \\
\hline
\end{tabular}

En los padres, a partir de 3 entradas, se obtuvieron 2 conglomerados con una calidad del conglomerado de 0,7 en la medida de silueta de cohesión y separación. Siendo el conglomerado Autoritativo del $87,0 \%$ (20) y el conglomerado Autoritario/Permisivo 13\% (3)

Tabla 2

Conglomerado bietápico Factores Robinson Padres

\begin{tabular}{lcccccc}
\hline \multirow{2}{*}{$\begin{array}{l}\text { Conglomerados } \\
\text { Robinson Padres }\end{array}$} & \multicolumn{2}{c}{$\begin{array}{c}\text { Factor Robinson } \\
\text { Autoritativo }\end{array}$} & \multicolumn{2}{c}{$\begin{array}{c}\text { Factor Robinson } \\
\text { Autoritario }\end{array}$} & \multicolumn{2}{c}{$\begin{array}{c}\text { Factor Robinson } \\
\text { Permisivo }\end{array}$} \\
& Media & DT & Media & DT & Media & DT \\
\hline Autoritativo & 4,27 &, 32 & 1,33 &, 29 & 1,8 &, 41 \\
Autoritario/Permisivo & 3,12 &, 08 & 2,18 &, 62 & 2,91 &, 17 \\
\hline
\end{tabular}

En las tablas 3 y 4 , se estudian la influencia del género de los padres sobre los estilos educativos y la implicación parental. En la tabla 3 padres y madres tienen puntuaciones significativamente diferentes en los estilos autoritario $(, 00)$ y permisivo $(, 00)$. 
Tabla 3

Padre/Madre y estilo educativo

\begin{tabular}{lcccccc}
\hline Factor Robinson & Sexo & $\mathrm{N}$ & Media & DT & $\mathrm{t}$ & Sig \\
\hline \multirow{2}{*}{ Autoritativo } & Padre & 23 & 4,13 &, 50 & \multirow{2}{*}{$, 1,40$} &, 16 \\
& Madre & 172 & 4,26 &, 42 & & \multirow{2}{*}{, 00} \\
\hline \multirow{2}{*}{ Autoritario } & Padre & 23 & 1,44 &, 44 & $-6,81$ &, 37 \\
\hline \multirow{2}{*}{ Permisivo } & Madre & 172 & 2,01 &, 53 & \multirow{2}{*}{3,60} & \\
& Padre & 23 & 1,97 &, 29 & & \\
\hline
\end{tabular}

En la tabla 4, no aparecen diferencias significativas entre padres y madres en las puntuaciones del cuestionario PTI.

Tabla 4

Padre/Madre implicación parental

\begin{tabular}{|c|c|c|c|c|c|c|}
\hline Factor PTI & Sexo & N & Media & DT & $\mathrm{t}$ & Sig \\
\hline \multirow{2}{*}{$\begin{array}{l}\text { Frecuencia de contacto entre la familia } \\
\text { y la escuela }\end{array}$} & Padre & 23 & 0,66 & ,49 & \multirow{2}{*}{, 61} & \multirow{2}{*}{, 55} \\
\hline & Madre & 172 & 0,60 & ,45 & & \\
\hline \multirow{2}{*}{$\begin{array}{l}\text { Calidad de las relaciones entre padres } \\
\text { y profesores }\end{array}$} & Padre & 23 & 3,01 &, 71 & \multirow{2}{*}{,- 94} & \multirow{2}{*}{,35 } \\
\hline & Madre & 172 & 3,13 & ,60 & & \\
\hline \multirow{2}{*}{$\begin{array}{l}\text { Implicación y voluntariado de los padres } \\
\text { en la escuela }\end{array}$} & Padre & 23 & 3,37 & ,63 & \multirow{2}{*}{.23} & \multirow{2}{*}{,82 } \\
\hline & Madre & 172 & 3,40 &, 58 & & \\
\hline \multirow{2}{*}{ Respaldo de los padres a los hijos } & Padre & 23 & 1,40 &, 49 & \multirow{2}{*}{,- 45} & \multirow{2}{*}{,65 } \\
\hline & Madre & 172 & 1,45 &, 48 & & \\
\hline
\end{tabular}

En la tabla 5 aparece la influencia de los estilos educativos sobre los factores de la implicación parental. En este caso, los estilos educativos no influyen significativamente en la implicación, aunque en el factor de apoyo de los padres a los hijos se aprecian tendencias de la influencia de los estilos educativos. 
Tabla 5

Estilos educativos con implicación parental

\begin{tabular}{|c|c|c|c|c|c|c|}
\hline Factor PTI & $\begin{array}{c}\text { Estilo } \\
\text { educativo }\end{array}$ & $\mathrm{N}$ & Media & DT & $\mathrm{t}$ & Sig. \\
\hline \multirow{2}{*}{$\begin{array}{l}\text { Frecuencia de contacto entre } \\
\text { la familia y la escuela }\end{array}$} & Autoritativo & 117 &, 60 & ,44 & \multirow[b]{2}{*}{,- 40} & \multirow[b]{2}{*}{, 69} \\
\hline & $\begin{array}{l}\text { Autoritario/ } \\
\text { Pasivo }\end{array}$ & 78 & ,63 &, 48 & & \\
\hline \multirow{2}{*}{$\begin{array}{l}\text { Calidad de las relaciones } \\
\text { entre padres y profesores }\end{array}$} & Autoritativo & 117 & 3,20 &, 60 & \multirow[b]{2}{*}{1,51} & \multirow[b]{2}{*}{, 13} \\
\hline & $\begin{array}{l}\text { Autoritario/ } \\
\text { Pasivo }\end{array}$ & 78 & 3,03 &, 61 & & \\
\hline \multirow{2}{*}{$\begin{array}{l}\text { Implicación y voluntariado } \\
\text { de los padres en la escuela }\end{array}$} & Autoritativo & 117 & 3,41 &, 61 & \multirow[b]{2}{*}{, 35} & \multirow{2}{*}{, 73} \\
\hline & $\begin{array}{l}\text { Autoritario/ } \\
\text { Pasivo }\end{array}$ & 78 & 3,38 &, 53 & & \\
\hline \multirow[b]{2}{*}{ Apoyo de los padres a los hijos } & Autoritativo & 117 & 1,50 &, 50 & \multirow[b]{2}{*}{1,81} & \multirow[b]{2}{*}{, 07} \\
\hline & $\begin{array}{l}\text { Autoritario/ } \\
\text { Pasivo }\end{array}$ & 78 & 1,37 & ,45 & & \\
\hline
\end{tabular}

En la tabla 6 encontramos las diferencias en la implicación parental en función del estilo educativo de padres y madres. En esta tabla se comparan 4 grupos (madres autoritativas, madres autoritarias/pasivas, padres autoritativos y padres autoritarios/pasivos) en función de sus puntuaciones en el cuestionario de implicación parental. En estas comparaciones no se han hallado diferencias significativas.

\section{Discusión}

Una vez mostrados los resultados es necesario recapitular para determinar su relación con los objetivos y las hipótesis planteadas en la introducción. Los resultados derivados de estudiar la influencia de los estilos educativos de padres y madres en la implicación parental son inexistentes porque las diferencias entre las variables no son significativas, por lo tanto la hipótesis 1 se rechaza.

La segunda hipótesis establece que las madres estarán más implicadas que los padres. Esta se rechaza porque no existen diferencias significativas en los factores de implicación entre padres y madres. No obstante, cuando comparamos los estilos educativos de padres y madres obtuvimos diferencias significativas en las puntuaciones de estilos autoritarios y permisivos. Las madres tuvieron puntuaciones más elevadas en referencia al estilo autoritario y los padres al permisivo. Un $88,2 \%$ de los cuestionarios fueron contestados por madres, esto sugiere que la participación predominante en la escuela es de las madres apoyando la afirmación hecha por Reay (1998) y Esquivel (1995) sugiriendo que las madres siguen siendo el principal vínculo entre la familia y la escuela.

El estudio se centra en los familiares de niños y niñas de primero de primaria por lo que la visión de la implicación parental está sesgada a un tiempo en concreto de la educación, por ello una futura línea de investigación podría ser repetir las mediciones en las diferentes etapas de la educación primaria. Debido a las diferencia muestral entre padres y madres que contestaron el cuestionario PTI, los resultados en la comparación entre el género y estilos de los padres pueden no ajustarse a la realidad convirtiéndose en una limitación por lo que una futura línea de investigación sería realizar la comparativa de género de los familiares con el mismo número de padres y madres. 


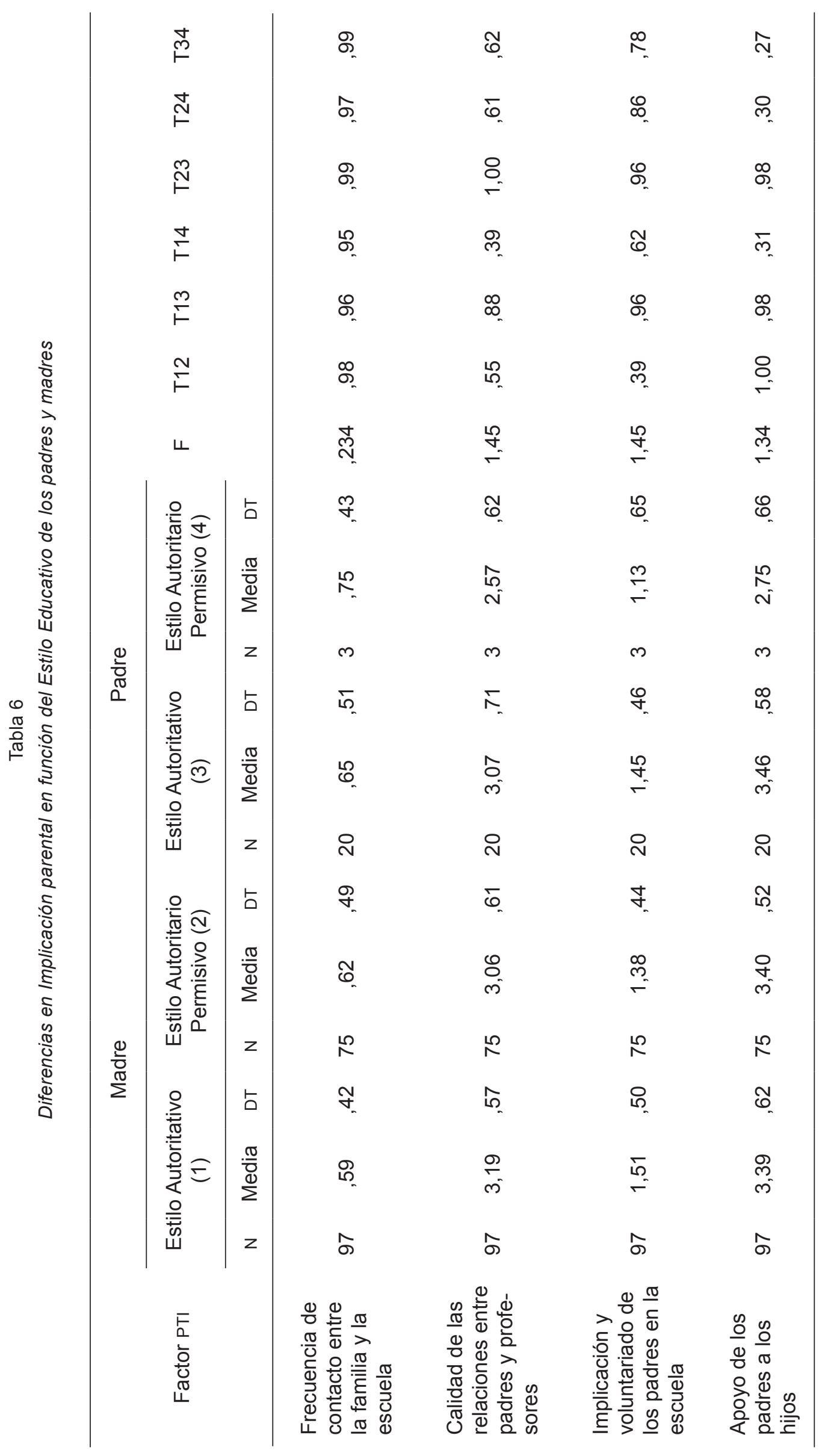




\section{Bibliografía}

Adams, K. S., y Christenson, S. L. (2000). Trust and the family-school relationship: An examination of parent-teacher differences in elementary and secondary grades. Journal of School Psychology, 38, 447-497.

Broderick, P.C., y Blewitt, P., (2006). The Life Span: Human Development for Helping Professionals, p.230.

Broderick P.C., y Blewitt P. (2012). «Four Parenting Styles,» education.com, http://www.education.com/reference/article/four-parenting-styles/

Comino, V. y Raya, T. (2014). Estilos educativos parentales y su relación con la socialización en adolescentes. Apuntes de Psicología, Vol. 32, 271-280.

Downey, D.B. (1994). The School Performance of Children from Single-mother and Single-father Families: Economic or Interpersonal Deprivation? Journal of Family Issues, 15, 129-147.

Epstein, J. L. (2001). School, family, and community partnerships: Preparing educators and improving schools. Boulder, CO: Westview Press.

Esquivel, L. (1995). Análisis de la tríada familia-escuela-sociedad: un estudio comparativo. Educación y Ciencia, 4, 51-62.

Grolnick, W. S., Kurowski, C. O., Dunlap, K. G., y Hevey, C. (2000). Parental resources and the transition to junior high. Journal of Research on Adolescence, 10, 465-488.

Harkness, S., Y Super, C. M. (1996). Parents' cultural belief systems: Their origins, expressions, and consequences. New York: Guilford.

Harris, K.M., Furstenberg, F. Jr., Y Marmer, J.K. (1998). Paternal Involvement with Adolescents in Intact Families: The Influence of Fathers Over the Life Course. Demography, 35, 201216.

Henderson, A. T. Y Mapp, K. L. (2002). A new wave of evidence: The impact of school, family, and community connections on student achievement. Austin, TX: Southwest Educational Development Laboratory.

Hoover-Dempsey, K. V., Walker, J. M. T., Sandler, H. M., Whetsel, D., Green, C. L., Wilkins, A. S., Y Closson, K. E. (2005). Why do parents become involved? Research findings and implications. Elementary School Journal, 106, 105-130.

Hornby, G. (2011) Parental Involvement in Childhood Education: Building Effective School-Family Partnerships. (1st ed.) New York: Springer.

Hsu, H-Y., Zhang, D., Kwok, 0-M., Li; Y., y Ju, S. (2011). Distinguishing the influences of father's and mother's involvement on adolescent academic achievement: Analyses of Taiwan Education Panel Survey data. Journal of Early Adolescence, 31, 694-713.

Ho, H-Z., Chen, W-W., Tran, C. N., y Ko, C-T. (2010). Parental involvement in Taiwanese families: Father-mother differences. Childhood Education, 86, 312-317.

Hung, C-L. (2005). Family background, parental involvement and environmental influences on Taiwanese children. The Alberta journal of Edw;ational Research, 51, 261-276.

Iruka, I. U., Winn, D. C., Kingsley, S. J., y Orthodoxou, Y. J. (2011). Links between parent teacher relationships and kindergartners' social skills: Do child ethnicity and family income matter? The Elementary School Journal, 111, 387-408.

John E. Beaulieu, y Alex Granzin, Working Parent Can Raise Smart Kids. (Tacoma, WA: Parkland Press, 1999), 115.

Keith, P. B., y Lichtman, M. V. (1994, December). Does parental involvement influence the academic achievement of Mexican-American eighth graders? Results from the National Education Longitudinal Study. School Psychology Quarterly, 9, 256- 273. 
Mattingly, D. J., Prislin, R., McKenzie, T. L., Rodriguez, J. L., y Kayzar, B. (2002). Evaluating evaluations: The case of parent involvement programs. Review of Educational Research, 72, 549-576.

McCaslin, M., y Murdock, T. (1991). The emergent interaction of home and school in the development of students' adaptive learning. In M. Maehr \& P. Pintrich (Eds.), Advances in motivation and achievement: Vol. 7. Motivationenhancing environments (pp. 213-259). Greenwich, CT: JAI.

National PTA, «Mission, Vision and Values. (2012) http://www.parentinvolvementmatters.org.

Nord, C.W., Brimhall, D., y West, J. (1997). Fathers' Involvement in their Children's Schools. NCES 98-091. Washington, DC: U.S. Department of Education, National Center for Education Statistics.

Pleck JH, Masciadrelli BP. Paternal involvement by U.S residential fathers: Levels, sources, and consequences. In: Lamb M, editor. The role of the father in child development. 4th. New York: John Wiley \& Sons; 2004. pp. 222-271.

Powell, D. R., Son, S., File, N, y San Juan, R. R. (2010). Parent-school relationships and children's academic and social outcomes in public school pre-kindergarten. Journal of School Psychology, 48, 269-292.

Reay, D. (1998) Class Work: Mothers' involvement in children's schooling London: University College Press.

Rimm-Kaufman, S. E., Pianta, R. C., Cox, M. J., y Bradley, R. H. (2003). Teacher-rated family involvement and children's social and academic outcomes in kindergarten. Early Education \& Development, 14, 179-198.

Rossi, A.S. (1978). A Biosocial Perspective on Parenting. In A.S. Rossi, J. Kagan, and T.K. Hareven, eds., The Family. New York: W.W. Norton \& Co., 1-31.

Serpell, Z. N., y Mashburn, A. J. (2011). Family-school connectedness and children's early social development. Social Development, 21, 21-46.

Shaver, A. V., y Walls, R. T. (1998). Effect of Title I parent involvement on student reading and mathematics achievement. Journal of Research and Development in Education, 31, 90-97.

Simon, B. S. (2004). High school outreach and family involvement. Social Psychology of Education, 7, 185-209.

Thomson, E., McLanahan, S.S., y Curtin, R.B. (1992). Family Structure, Gender, and Parental Socialization. Journal of Marriage and the Family, 54, 368-378.

Yamamura, E. K., Martinez, M. A., y Saenz, V. B. (2010). Moving beyond high school expectations: Examining stakeholders' responsibility for increasing Latina/o students' college readiness. The High School Journal, 93, 126-148.

Zablotsky, B., Boswell, K., y Smith, C. (2012). An evaluation of school involvement and satisfaction of parents of children with autism spectrum disorders. American Journal on Intellectual and Developmental Disabilities, 117, 316-330. 